\title{
Spacecraft Attitude Synchronization And Formation Keeping Using Line Of Sight Measurements
}

\author{
Rakesh R. Warier* Arpita Sinha* Srikant Sukumar* \\ * Indian Institute of Technology, Bombay, India (e-mail: \\ \{rakeshwarier,asinha,srikant\}@sc.iitb.ac.in).
}

\begin{abstract}
:
This paper considers the problem of attitude synchronization and formation keeping of two spacecrafts, with a leader. Attitudes of spacecrafts are not measured directly, instead control torques are determined from the line-of-sight (LOS) vectors between two spacecrafts, and another set of LOS vectors from a common leader. We provide distributed position and attitude control laws. Attitude control law is proposed to ensure that the two follower spacecrafts reach attitude consensus. A formation keeping position control law is also proposed so that desired distance between two follower spacecrafts and each spacecraft to the leader is achieved. Proposed position control law uses relative velocities and LOS vectors in the respective body frames of the two spacecrafts. The state feedback laws proposed in this work guarantee almost semi-global asymptotic stability of the desired closed-loop equilibrium.
\end{abstract}

\section{INTRODUCTION}

Controlling the attitude or the orientation of a satellite is very important since satellites have equipment that need to be pointed in desired directions. Spacecraft attitude dynamics is usually modelled as rigid body dynamics and can be decoupled from the translation dynamics of the spacecraft. Even for a single rigid body the control of attitude dynamics is highly non-linear problem.

Recently, the idea of using multiple satellites cooperatively working together to achieve a common mission has received considerable attention. The main attraction of the idea is that a group of satellites flying as a formation can act as one large virtual instrument which will be more capable and robust than a monolithic satellite. Also building and deploying multiple small satellites is cheaper than that of larger single satellite of same combined weight. Satellites in formation flying missions are however required to work together, implying that they need to maintain some relative attitude and position relative to each other. These precise attitude and position maintenance requirements pose new control challenges. Further, constraints on communication bandwidth render centralized control almost impossible in real space applications. The focus of formation flying research is in designing cooperative control laws for a group of autonomous satellites.

\subsection{Related Works}

There are many articles in literature that make use of LOS unit vector measurements for relative navigation or relative orbit determination of spacecraft. The idea of inertial LOS based cyclic formation control is proposed in Gurfill et al [2007]. Relative orbit determination using LOS vectors is considered in Woffinden et al [2009], to cite a few. The idea of using LOS unit vectors in body frames to directly control attitude is more recent. Andrle et al [2009] shows that deterministic relative attitude determination is possible for a formation of three spacecrafts. Lee [2012] proposes a control law to asymptotically stabilize relative attitude between two spacecrafts, making use of line of sight (LOS) observations between them and LOS observations to a common object. However in Lee [2012] the relative positions of two spacecraft and the common object are assumed to be fixed. We consider the combined problem of attitude synchronization and formation keeping of two spacecraft and hence the relative positions of spacecraft are not fixed. Warier et al [2013] considers formation keeping and attitude alignment with out the common object, but attitude alignment is only along line joining two spacecrafts. Even after considering combined position and attitude dynamics, our attitude synchronization is shown to be independent of position dynamics as long as the three spacecrafts are not collinear or coinciding.

\section{PROBLEM FORMULATION}

We consider complete attitude synchronization and formation keeping of two spacecraft making use of LOS measurements to a leader spacecraft and to each other in the respective body frames. The reference spacecraft is assumed to have constant velocity. We describe some of the mathematical preliminaries in the next section and then describe the dynamics of the problem considered.

\subsection{Mathematical Preliminaries}

Attitude of a rigid body represented as a rotational matrix forms a compact manifold, given by

$$
S O(3)=\left\{R \in \mathbb{R}^{3 \times 3} \mid R^{\top} R=R R^{\top}=I, \operatorname{det}(R)=1\right\}
$$


$S O(3)$ forms a Lie group under the group operation matrix multiplication. Lie algebra of $S O(3)$ is denoted as $s o(3)$ and is given by

$$
\text { so }(3)=\left\{S \in \mathbb{R}^{3 \times 3} \mid S=-S^{\top}\right\}
$$

Map $\wedge: \mathbb{R}^{3} \rightarrow s o(3)$ denotes the isomorphism from $\mathbb{R}^{3}$ to so(3). If $x=\left[x_{1}, x_{2}, x_{3}\right]^{T}, x \in \mathbb{R}^{3}$

$$
\hat{x}=\left[\begin{array}{ccc}
0 & -x_{3} & x_{2} \\
x_{3} & 0 & -x_{1} \\
-x_{2} & x_{1} & 0
\end{array}\right]
$$

Further $\hat{x}$ represents the skew symmetric matrix implementing cross product, i.e. $\hat{x} y=x \times y, \forall y$. It's inverse is denoted as $\vee: s o(3) \rightarrow \mathbb{R}^{3}$, implicitly we can define $(\hat{x})^{\vee}=x$. We define a map $\mathcal{S}: \mathbb{R}^{3 \times 3} \rightarrow$ so $(3)$, as $\mathcal{S}(A):=\frac{A-A^{\top}}{2}$. This map $\mathcal{S}$ has set of skew symmetric matrices as its range.

The following results are useful. Let $x \in \mathbb{R}^{n}$, then we have 2-norm of $x$ is defined as $\|x\|=\sqrt{x \cdot x}$

$$
\frac{d}{d t}\|x\|=\frac{d}{d t} \sqrt{x \cdot x}=\frac{1}{2 \sqrt{x \cdot x}} 2 x \cdot \dot{x}=\frac{x \cdot \dot{x}}{\|x\|}
$$

$\forall a_{1}, a_{2} \in \mathbb{R}^{3}$, and $R \in \mathrm{SO}(3)$,

$$
a_{1} \cdot a_{2}=\left(R a_{1}\right) \cdot\left(R a_{2}\right)
$$

$\operatorname{tr}()$ is the trace of a square matrix, defined as sum of its diagonal elements, $\forall A_{1}, A_{2} \in R^{n \times n}$

$$
\operatorname{tr}\left(A_{1}^{\top} A_{2}\right)=\operatorname{tr}\left(A_{2}^{\top} A_{1}\right)=\operatorname{tr}\left(A_{1} A_{2}^{\top}\right)
$$

\subsection{Dynamics}

The attitude of a spacecraft is the orientation of its body fixed frame with respect to the inertial reference frame. Equations of motion of the attitudes of $i$-th spacecraft, for $i=1,2$ are given by

$$
\begin{aligned}
\dot{R}_{i} & =R_{i} \hat{\Omega}_{i} \\
J_{i} \dot{\Omega}_{i} & =J_{i} \Omega_{i} \times \Omega_{i}+\tau_{i}
\end{aligned}
$$

where $J_{i} \in \mathbb{R}^{3 \times 3}$ is the moment of inertia, $\Omega_{i} \in \mathbb{R}^{3}$ the angular velocity, and $\tau_{i} \in \mathbb{R}^{3}$ the control torque in of $i$-th spacecraft in its body fixed frame.

Translational dynamics of centre of mass of the spacecraft is assumed to be double integrator dynamics. For the $i$-th spacecraft we have

$$
\begin{aligned}
& m_{i} \dot{r}_{i}=m_{i} v_{i} \\
& m_{i} \dot{v}_{i}=f_{i}
\end{aligned}
$$

where $m_{i} \in \mathbb{R}, m_{i}>0$, is the mass of the spacecraft and $r_{i}, v_{i} \in \mathbb{R}^{3}$ are the position and the velocity of the spacecraft in the inertial frame. $f_{i} \in \mathbb{R}^{3}$ is the force applied on the $i$-th spacecraft represented in the inertial frame. We take

$$
u_{i}=\frac{f_{i}}{m_{i}}
$$

where $u_{i} \in \mathbb{R}^{3}$ represents the translation control input for the spacecraft in the inertial frame And the dynamics of the reference spacecraft is given by

$$
\begin{aligned}
& \dot{r}_{3}=v_{3} \\
& \dot{v}_{3}=0
\end{aligned}
$$

\subsection{Measured Variables}

We assume that the spacecrafts lack a common frame of reference and do not make any absolute measurements. The spacecrafts 1 and 2 make relative measurements between each other and to the leader, about their body fixed frames, and communicate the same to each other. Leader does not communicate with the follower spacecrafts.

Let $(i, j) \in\{(1,2),(2,1),(1,3),(2,3)\}$. We denote the line of sight unit vector observed from the $i$-th spacecraft to the $j$-th spacecraft and represented in the inertial frame as $s_{i j}$. This is given by

$$
s_{i j}=\frac{\left(r_{j}-r_{i}\right)}{\left\|\left(r_{j}-r_{i}\right)\right\|}
$$

Measurements are however made in each spacecraft's own body frame. We define $l_{i j}$ as the line of sight unit vector observed from the $i$-th spacecraft to the $j$-th spacecraft, represented in the $i$-th body fixed frame. This is given by

$$
l_{i j}=R_{i}^{\top} s_{i j}=R_{i}^{\top} \frac{\left(r_{j}-r_{i}\right)}{\left\|\left(r_{j}-r_{i}\right)\right\|}
$$

In addition to LOS unit vectors each spacecraft measures relative velocity with respect to each other and with respect to the leader. We define $v_{i j}$ as relative velocity of the $j$-th spacecraft observed from the $i$-th spacecraft given by

$$
v_{i j}=R_{i}^{\top}\left(v_{j}-v_{i}\right)
$$

Distance between $i$-th and $j$-th spacecraft is given by

$$
d_{i j}=\left\|r_{j}-r_{i}\right\|
$$

\subsection{Control Objectives}

We consider two spacecrafts with dynamics given in (7)(10) along with leader spacecraft dynamics given by (12)(13) which are able to communicate LOS unit vectors with each other. The control objectives are to achieve,

(1) Formation Keeping $\left\|r_{1}-r_{2}\right\|=d_{12},\left\|r_{1}-r_{3}\right\|=d_{13}^{s}$ and $\left\|r_{3}-r_{2}\right\|=d_{23}^{s}$, where $d_{12}^{s}, d_{13}^{s}$ and $d_{23}^{s}$ ae the desired distances between the three spacecrafts.

(2) Velocity synchronization, $v_{1}=v_{2}=v_{3}$

(3) Attitude synchronization $R_{1}=R_{2}$, and $\Omega_{1}=\Omega_{2}=0$ asymptotically. The control problem is considered under the following assumptions,

(A1) The centre of masses of the three spacecraft are not collinear or coinciding $\left(r_{2}(t)-r_{1}(t)\right) \times\left(r_{3}(t)-r_{2}(t)\right) \neq$ $\left[\begin{array}{lll}0 & 0 & 0\end{array}\right]^{\top}, \forall t>0$.

(A2) The desired distances between spacecraft, $\left.d_{12}^{s}, d_{13}^{s}\right]$ and $d_{23}^{s}$ are feasible for a triangle i.e. satisfies the triangle inequalities.

$$
d_{12}^{s}<d_{13}^{s}+d_{23}^{s} \quad d_{13}^{s}<d_{12}^{s}+d_{23}^{s} \quad d_{23}^{s}<d_{12}^{s}+d_{13}^{s}
$$

\section{RELATIVE ATTITUDE DETERMINATION}

We make use of LOS vectors measured in respective body frames, $l_{12}, l_{21}, l_{13}$ and $l_{23}$ to determine relative attitude. We refer to Wertz [1978].

$$
\begin{aligned}
& l_{123}:=\frac{\left(l_{12} \times l_{13}\right)}{\left\|l_{12} \times l_{13}\right\|}=\frac{R_{1}^{\top}\left(\left(r_{2}-r_{1}\right) \times\left(r_{3}-r_{1}\right)\right)}{\left\|\left(r_{2}-r_{1}\right) \times\left(r_{3}-r_{2}\right)\right\|} \\
& l_{213}:=\frac{\left(l_{21} \times l_{23}\right)}{\left\|l_{21} \times l_{23}\right\|}=\frac{R_{2}^{\top}\left(\left(r_{1}-r_{2}\right) \times\left(r_{3}-r_{2}\right)\right)}{\left\|\left(r_{1}-r_{2}\right) \times\left(r_{3}-r_{2}\right)\right\|}
\end{aligned}
$$


In addition we have

$$
\begin{aligned}
\left(r_{2}-r_{1}\right) \times\left(r_{3}-r_{1}\right) & =\left(r_{2}-r_{3}+r_{3}-r_{1}\right) \times\left(r_{3}-r_{1}\right) \\
& =\left(r_{3}-r_{1}\right) \times\left(r_{3}-r_{2}\right) \\
\left(r_{1}-r_{2}\right) \times\left(r_{3}-r_{2}\right) & =\left(r_{1}-r_{3}+r_{3}-r_{2}\right) \times\left(r_{3}-r_{2}\right) \\
& =-\left(r_{3}-r_{1}\right) \times\left(r_{3}-r_{2}\right)
\end{aligned}
$$

Since spacecrafts are assumed to be non collinear, we have $\left(r_{3}-r_{1}\right) \times\left(r_{3}-r_{2}\right) \neq\left[\begin{array}{ll}0 & 0\end{array}\right]^{\top}$ and $l_{123}$ and $l_{213}$ are well defined. If we define $s_{123}=\frac{\left(r_{3}-r_{1}\right) \times\left(r_{3}-r_{2}\right)}{\left\|\left(r_{3}-r_{1}\right) \times\left(r_{3}-r_{2}\right)\right\|}$, then $l_{123}=R_{1}^{\top}\left(s_{123}\right)$ and $l_{213}=-R_{2}^{\top}\left(s_{123}\right)$, Observe that $s_{123}=s_{12} \times s_{13}$.

Construct a matrix with unit vectors $l_{12}, l_{123}$ and $l_{12} \times l_{123}$ as the column vectors.

$$
\begin{aligned}
P_{1} & :=\left[l_{12}\left(l_{123}\right)\left(l_{12} \times l_{123}\right)\right] \\
& =\left[R_{1}^{\top} s_{12} R_{1}^{\top}\left(s_{123}\right) R_{1}^{\top}\left(s_{12} \times s_{123}\right)\right] \\
& =R_{1}^{\top}\left[s_{12}\left(s_{123}\right)\left(s_{12} \times s_{123}\right)\right]
\end{aligned}
$$

Similarly we can construct a matrix with unit vectors $l_{21}$, $l_{213}$ and $\left(l_{21} \times l_{213}\right)$ as the column vectors

$$
\begin{aligned}
P_{2} & :=\left[l_{21}\left(l_{213}\right)\left(l_{213}\right) \times l_{21}\right] \\
& =-\left[R_{2}^{\top} s_{12} R_{2}^{\top}\left(s_{123}\right) R_{2}^{\top}\left(s_{12} \times s_{123}\right)\right] \\
& =-R_{2}^{\top}\left[s_{12}\left(s_{123}\right)\left(s_{12} \times s_{123}\right)\right]
\end{aligned}
$$

Note that columns vectors of both $P_{1}$ and $P_{2}$ form orthogonal bases, and thus $P_{1}$ and $P_{2}$ are orthogonal matrices. We get

$$
\left[s_{12}\left(s_{123}\right)\left(s_{12} \times s_{123}\right)\right]=-R_{2} P_{2}=R_{1} P_{1}
$$

We can determine the relative attitude $Q:=R_{1}^{\top} R_{2}$ using (23), to be

$$
Q=R_{1}^{\top} R_{2}=-P_{1} P_{2}^{\top}
$$

The attitude determination scheme is not explicitly used in the control law. We make use of the scheme to show that our control law indeed obtains attitude synchronization.

\section{ERROR FUNCTIONS}

We make use of error functions to design the control law. Error functions are chosen such that minimizing of error functions will achieve the control objectives.

\subsection{Attitude Error Function}

Trace and modified trace functions are very commonly used in attitude control design. Some examples from the literature include Chaturvedi et al. [2011], Sarlette et al. [2009] and Nair et al [2007]. For $R_{1}, R_{2} \in S O(3)$, $\operatorname{tr}\left(R_{1}^{\top} R_{2}\right)=1+2 \cos (\theta)$, where $\theta$ is the angle of single axis rotation between $R_{1}$ and $R_{2} \cdot \operatorname{tr}\left(R_{1}^{T} R_{2}\right)$ obtains maximum value when orientation of both satellites are identical, i.e. $R_{1}=R_{2}$. Let

$$
\Psi_{1}\left(R_{1}, R_{2}\right)=\left(\operatorname{tr}\left(I-R_{1}^{\top} R_{2}\right)\right)
$$

The derivative of $\Psi_{1}$ turns out to be,

$$
\frac{d \Psi_{1}}{d t}=-2\left(\mathcal{S}\left(R_{1}^{\top} R_{2}\right)\right)^{\vee} \cdot \Omega_{1}-2\left(\mathcal{S}\left(R_{2}^{\top} R_{1}\right)\right)^{\vee} \cdot \Omega_{2}
$$

The proof is available in Sarlette [2009b]. The critical points of function $\Psi_{1}$ are obtained by solving

$$
\left(\mathcal{S}\left(R_{1}^{\top} R_{2}\right)\right)^{\vee}=0
$$

which implies $\left(R_{1}^{\top} R_{2}\right)^{\top}=\left(R_{1}^{\top} R_{2}\right)$, both matrices orthogonal (with determinant +1 ) and symmetric, which gives us that

$$
R_{1}^{\top} R_{2} \in\{I, \operatorname{diag}[1,-1,-1], \quad \operatorname{diag}[-1,-1,1],
$$

Also observe that at $I$, the trace function is at its minimum and at other critical points it achieves the maximum value of 4 .

It is interesting to see $\Psi_{1}$ in terms of the attitude determination scheme (24)

$$
\begin{aligned}
\Psi_{1}= & \operatorname{tr}\left(I-R_{1}^{\top} R_{2}\right)=3+\operatorname{tr}\left(P_{1} P_{2}^{\top}\right)=3+\operatorname{tr}\left(P_{1}^{\top} P_{2}\right) \\
= & 3+l_{12} \cdot l_{21}+l_{123} \cdot l_{213}-\left(l_{12} \times l_{123}\right) \cdot\left(l_{21} \times l_{213}\right) \\
= & \left(1+l_{12} \cdot l_{21}\right)+\left(1+l_{123} \cdot l_{213}\right) \\
& +\left(1-\left(l_{12} \times l_{123}\right) \cdot\left(l_{21} \times l_{213}\right)\right)
\end{aligned}
$$

Expression of $\Psi_{1}$ is obtained in terms of LOS unit vectors. This is desirable for controlling attitude using LOS unit vectors. In Warier et al [2013], the attitude alignment error function is $\left(1+l_{12} \cdot l_{21}\right)$. In Lee [2012], the error functions used are of linear combination of $\left(1+l_{12} \cdot l_{21}\right)$ and $\left(1+l_{123} \cdot l_{213}\right)$. It can be seen that our expression has additional term compared to Lee [2012].

\subsection{Distance error function}

We define a distance error functions of the form

$$
\begin{aligned}
\Psi_{12}^{d} & =\frac{1}{2}\left(\left\|r_{1}-r_{2}\right\|-d_{12}^{s}\right)^{2} \\
\Psi_{13}^{d} & =\frac{1}{2}\left(\left\|r_{1}-r_{3}\right\|-d_{13}^{s}\right)^{2} \\
\Psi_{23}^{d} & =\frac{1}{2}\left(\left\|r_{2}-r_{3}\right\|-d_{23}^{s}\right)^{2}
\end{aligned}
$$

We can see that the derivatives of the distance error function are,

$$
\begin{aligned}
& \dot{\Psi}_{12}^{d}=\frac{\left(d_{12}-d_{12}^{s}\right)}{2}\left(l_{12} \cdot v_{12}+l_{21} \cdot v_{21}\right) \\
& \dot{\Psi}_{13}^{d}=\left(d_{13}-d_{13}^{s}\right)\left(l_{13} \cdot v_{13}\right) \\
& \dot{\Psi}_{23}^{d}=\left(d_{23}-d_{23}^{s}\right)\left(l_{23} \cdot v_{23}\right)
\end{aligned}
$$

(4) was used to get the expressions.

\subsection{Velocity synchronisation error function}

We define a velocity synchronization error function

$$
\Psi^{v}\left(v_{1}, v_{2}, v_{3}\right)=\frac{1}{2}\left(\left\|v_{3}-v_{1}\right\|^{2}+\left\|v_{3}-v_{2}\right\|^{2}\right)
$$

with derivative

$$
\begin{aligned}
\dot{\Psi^{v}} & =-\left(v_{3}-v_{1}\right) \cdot\left(u_{1}\right)-\left(v_{3}-v_{2}\right) \cdot\left(u_{2}\right) \\
& =-\left(v_{13}\right) \cdot\left(R_{1}^{\top} u_{1}\right)-\left(v_{23}\right) \cdot\left(R_{2}^{\top} u_{2}\right)
\end{aligned}
$$

\section{COMBINED ATTITUDE AND POSITION CONTROL}

Lemma 1. Let $A, B \in \mathbb{R}^{3 \times 3}$, Then

$\left(2 \mathcal{S}\left(A B^{\top}\right)\right)^{\vee}=\left(A B^{\top}-B A^{\top}\right)^{\vee}=-(a \times x)-(b \times y)-(c \times z)$

where $a, b, c$ are column vectors of $A$ and $x, y, z$ column vectors of $B$. 
Proof. Let $\Gamma=\left(A B^{\top}-B A^{\top}\right)$. Clearly $\Gamma$ is skew symmetric, thus has diagonal elements zero. Non diagonal elements of $\Gamma$ are obtained to be

$$
\begin{aligned}
(\Gamma)_{12}= & \left(a_{1} x_{2}-a_{2} x_{1}\right)+\left(b_{1} y_{2}-b_{2} y_{1}\right) \\
& +\left(c_{1} z_{2}-c_{2} z_{1}\right) \\
(\Gamma)_{13}= & \left(a_{1} x_{3}-a_{3} x_{1}\right)+\left(b_{1} y_{3}-b_{3} y_{1}\right) \\
& +\left(c_{1} z_{3}-c_{3} z_{1}\right) \\
(\Gamma)_{23}= & \left(a_{2} x_{3}-a_{3} x_{2}\right)+\left(b_{2} y_{3}-b_{3} y_{2}\right) \\
& +\left(c_{2} z_{3}-c_{3} z_{2}\right)
\end{aligned}
$$

And by definition of $\vee$ map we have

$$
(\Gamma)^{\vee}=\left[\begin{array}{c}
-(\Gamma)_{23} \\
(\Gamma)_{13} \\
-(\Gamma)_{12}
\end{array}\right]
$$

Now notice the standard expression of vector product given by,

$$
\left[\begin{array}{l}
a_{1} \\
a_{2} \\
a_{3}
\end{array}\right] \times\left[\begin{array}{l}
x_{1} \\
x_{2} \\
x_{3}
\end{array}\right]=\left[\begin{array}{c}
a_{2} x_{3}-a_{3} x_{2} \\
-\left(a_{1} x_{3}-a_{3} x_{1}\right) \\
a_{1} x_{2}-a_{2} x_{1}
\end{array}\right]
$$

Substituting (38)-(40) in (41) and comparing terms with (42), the identity (37) is evident.

\subsection{Control Law}

$$
\begin{aligned}
\tau_{1}= & -k_{\Omega_{1}} \Omega_{1}+\frac{k_{1}}{\gamma}\left(l_{12} \times l_{21}+l_{123} \times l_{213}\right) \\
& -\frac{k_{1}}{\gamma}\left(\left(l_{12} \times l_{123}\right) \times\left(l_{21} \times l_{213}\right)\right) \\
\tau_{2}= & -k_{\Omega_{2}} \Omega_{2}+\frac{k_{1}}{\gamma}\left(l_{21} \times l_{12}+l_{213} \times l_{123}\right) \\
& -\frac{k_{1}}{\gamma}\left(\left(l_{21} \times l_{213}\right) \times\left(l_{12} \times l_{123}\right)\right) \\
R_{1}^{\top} u_{1}= & k_{v_{1}} v_{13}+k_{d_{1}}\left(d_{12}-d_{12}^{s}\right) l_{12}+k_{d_{2}}\left(d_{13}-d_{13}^{s}\right) l_{13} \\
R_{2}^{\top} u_{2}= & k_{v_{2}} v_{23}+k_{d_{1}}\left(d_{12}-d_{12}^{s}\right) l_{21}+k_{d_{3}}\left(d_{23}-d_{23}^{s}\right) l_{23}
\end{aligned}
$$

where the control gains

$$
k_{\Omega_{1}}, k_{\Omega_{2}}, k_{v_{1}}, k_{v_{2}}, k_{1}, k_{d_{1}}, k_{d_{2}}, k_{d_{3}}, \gamma>0
$$

Here $\gamma$ is chosen depending on initial conditions so as to ensure asymptotic convergence to the desired equilibrium as follows

$$
\begin{gathered}
4 k_{1}-k_{1} \Psi_{1}(0)<\gamma\left(\sum_{i=1}^{2} \lambda_{\max _{i}}\left(J_{i}\right)\left\|\Omega_{i}(0)\right\|^{2}+\Psi^{v}(0)\right) \\
+\gamma\left(k_{d_{1}} \Psi_{12}^{d}(0)+k_{d_{2}} \Psi_{13}^{d}(0)+k_{d_{3}} \Psi_{23}^{d}(0)\right)
\end{gathered}
$$

Since we assume that the two spacecraft have no access to inertial coordinates, the control needs to be expressed in the local frame. Position control input $u_{i}$ expressed in $i$-th spacecraft's body frame is $R_{i}^{\top} u_{i}$.

\subsection{Stability Results}

Proposition 2. Consider the system dynamics given by (7)-(10) under the assumptions (A1)-(A2) and control law given by (43)-(46)

(i) The system has two equilibrium configurations.

$$
\begin{gathered}
\mathcal{M}_{1}=\left\{\left(R_{1}, \Omega_{1}, R_{2}, \Omega_{2}, r_{1}, r_{2}, v_{1}, v_{2}\right) \mid R_{1}=R_{2},\right. \\
\Omega_{1}=\Omega_{2}=0, v_{1}=v_{2}=v_{3},\left\|r_{1}-r_{2}\right\|=d_{12}^{s} \\
\left.\left\|r_{1}-r_{3}\right\|=d_{13}^{s},\left\|r_{2}-r_{3}\right\|=d_{23}^{s}\right\} \\
\mathcal{M}_{2}=\left\{\left(R_{1}, \Omega_{1}, R_{2}, \Omega_{2}, r_{1}, r_{2}, v_{1}, v_{2}\right) \mid R_{1}^{\top} R_{2} \in \mathcal{N},\right. \\
\Omega_{1}=\Omega_{2}=0, v_{1}=v_{2}=v_{3},\left\|r_{1}-r_{2}\right\|=d_{12}^{s}, \\
\left.\left\|r_{1}-r_{3}\right\|=d_{13}^{s},\left\|r_{2}-r_{3}\right\|=d_{23}^{s}\right\}
\end{gathered}
$$

where

$\mathcal{N}=\{\operatorname{diag}[1,-1,-1], \operatorname{diag}[-1,-1,1], \operatorname{diag}[-1,1,-1]\}$ and $\mathcal{M}_{1}, \mathcal{M}_{2} \in T S O(3) \times T S O(3) \times \mathbb{R}^{12}$

(ii) Desired equilibrium configuration $\mathcal{M}_{1}$ is asymptotically stable and a conservative region of attraction of $\mathcal{M}_{1}$ is almost semi global, i.e. region of attraction of $\mathcal{M}_{1}$ can be increased to include the entire space, except the undesirable equilibrium configurations

(iii) Undesired equilibrium configuration $\mathcal{M}_{2}$ is unstable

Proof. We make use of the La Salle's invariance principle and Chetaev's instability theorem for the proof. We define new variables

$$
\begin{array}{ll}
\mathcal{X}:=R_{1}^{\top} R_{2} & \\
\xi_{1}:=r_{3}-r_{1} & \xi_{2}=r_{3}-r_{2} \\
\zeta_{1}:=v_{3}-v_{1} & \zeta_{2}=v_{3}-v_{2}
\end{array}
$$

Under new variables $\left(\mathcal{X}, \Omega_{1}, \Omega_{2}, \xi_{1}, \zeta_{1}, \xi_{2}, \zeta_{2}\right)$, the system dynamics can be written as

$$
\begin{aligned}
\dot{\mathcal{X}} & =\mathcal{X} \hat{\Omega}_{2}-\hat{\Omega}_{1} \mathcal{X} \\
J_{i} \dot{\Omega}_{i} & =J_{i} \Omega_{i} \times \Omega_{i}+\tau_{i} \\
\dot{\xi}_{i} & =\zeta_{i} \\
\dot{\zeta}_{i} & =-u_{i}
\end{aligned}
$$

where $i=1,2$.

(i) Consider the Lyapunov like function consisting of $\Psi_{1}$, $\Psi_{12}^{d}, \Psi_{13}^{d}, \Psi_{23}^{d}$ and $\Psi^{v}$.

$$
\begin{aligned}
\mathcal{V}= & k_{1} \Psi_{1}+\frac{\gamma}{2} \Omega_{1} \cdot\left(J_{1} \Omega_{1}\right)+\frac{\gamma}{2} \Omega_{2} \cdot\left(J_{2} \Omega_{2}\right) \\
& +\gamma k_{d_{1}} \Psi_{12}^{d}+\gamma k_{d_{2}} \Psi_{13}^{d}+\gamma k_{d_{3}} \Psi_{23}^{d}+\gamma \Psi^{v}
\end{aligned}
$$

In terms of changed variables we have

$$
\begin{aligned}
\mathcal{V}= & k_{1} \operatorname{tr}(I-\mathcal{X})+\frac{\gamma}{2} \Omega_{1} \cdot\left(J_{1} \Omega_{1}\right)+\frac{\gamma}{2} \Omega_{1} \cdot\left(J_{2} \Omega_{2}\right) \\
& +\frac{\gamma k_{d_{1}}}{2}\left(\left\|\xi_{1}-\xi_{2}\right\|-d_{12}^{s}\right)^{2}+\frac{\gamma k_{d_{2}}}{2}\left(\left\|\xi_{1}\right\|-d_{13}^{s}\right)^{2} \\
& +\frac{\gamma k_{d_{13}}}{2}\left(\left\|\xi_{2}\right\|-d_{23}^{s}\right)^{2}+\frac{\gamma}{2}\left(\left\|\zeta_{1}\right\|^{2}+\left\|\zeta_{2}\right\|^{2}\right)
\end{aligned}
$$

Clearly $\mathcal{V} \geq 0$, and $\mathcal{V}=0$ only when the desired control objectives are satisfied. $\mathcal{V}$ is positive definite with respect to variables $I-\mathcal{X}, \Omega_{1}, \Omega_{2},\left\|\xi_{1}\right\|-d_{13}^{s}$, $\left\|\xi_{2}\right\|-d_{23}^{s},\left\|\xi_{1}-\xi_{2}\right\|-d_{12}^{s}, \zeta_{1}$ and $\left\|\zeta_{2}\right\|$.

$$
\begin{aligned}
& \mathcal{X}=I, \text { i.e. } R_{1}=R_{2}, \Omega_{1}=\Omega_{2}=0, \\
& \left\|r_{3}-r_{1}\right\|=\left\|\xi_{1}\right\|=d_{13}^{s} \\
& \left\|r_{3}-r_{2}\right\|=\left\|\xi_{2}\right\|=d_{23}^{s} \\
& \left\|r_{1}-r_{2}\right\|=\left\|\xi_{1}-\xi_{2}\right\|=d_{12}^{s}, \zeta_{1}=\zeta_{2}=0
\end{aligned}
$$

If the changed variables $\left(\mathcal{X}, \Omega_{1}, \Omega_{2}, \xi_{1}, \zeta_{1}, \xi_{2}, \zeta_{2}\right)$ satisfy conditions (55)-(58), we have the original variables $\left(R_{1}, \Omega_{1}, R_{2}, \Omega_{2}, r_{1}, v_{1}, r_{2}, v_{2}\right) \in \mathcal{M}_{1}$. Now taking the derivative we have,

$$
\begin{aligned}
\dot{\mathcal{V}}= & k_{1} \dot{\Psi}_{1}+\gamma \Omega_{1} \cdot \tau_{1}+\gamma \Omega_{2} \cdot \tau_{2}+ \\
& +\gamma k_{d_{1}} \dot{\Psi}_{12}^{d}+\gamma k_{d_{2}} \dot{\Psi}_{13}^{d}+\gamma k_{d_{3}} \dot{\Psi}_{23}^{d}+\gamma \dot{\Psi}^{v}
\end{aligned}
$$


Substituting derivatives of error functions,

$$
\begin{aligned}
\dot{\mathcal{V}}= & -2 k_{1}\left(\mathcal{S}\left(R_{1}^{\top} R_{2}\right)\right)^{\vee} \cdot \Omega_{1}-2 k_{1}\left(\mathcal{S}\left(R_{2}^{\top} R_{1}\right)\right)^{\vee} \cdot \Omega_{2} \\
& +\gamma \Omega_{1} \cdot \tau_{1}+\gamma \Omega_{2} \cdot \tau_{2}+\frac{k_{d_{1}} \gamma\left(d_{12}-d_{12}^{s}\right)}{2}\left(l_{12} \cdot v_{12}\right) \\
& +k_{d_{1}} \gamma \frac{\left(d_{12}-d_{12}^{s}\right)}{2}\left(l_{21} \cdot v_{21}\right) \\
& +k_{d_{2}} \gamma\left(d_{13}-d_{13}^{s}\right)\left(l_{13} \cdot v_{13}\right) \\
& +k_{d_{3}} \gamma\left(d_{23}-d_{23}^{s}\right)\left(l_{23} \cdot v_{23}\right) \\
& -\gamma\left(v_{13}\right) \cdot\left(R_{1}^{\top} u_{1}\right)-\gamma\left(v_{23}\right) \cdot\left(R_{2}^{\top} u_{2}\right)
\end{aligned}
$$

By the attitude determination scheme, $Q=$ $P_{1} P_{2}^{\top}=R_{1}^{\top} R_{2}$. Now making use of (37), we have

$$
\begin{aligned}
\left(2 \mathcal{S}\left(R_{1}^{\top} R_{2}\right)\right)^{\vee}= & \left(-l_{12} \times l_{21}-l_{123} \times l_{213}\right) \\
& +\left(l_{12} \times l_{123}\right) \times\left(l_{21} \times l_{213}\right)
\end{aligned}
$$

Substituting control terms from (43)-(46) and using (59) we obtain,

$$
\begin{aligned}
\dot{\mathcal{V}}= & -\gamma\left(k_{\Omega_{1}}\left\|\Omega_{1}\right\|^{2}+k_{\Omega_{2}}\left\|\Omega_{2}\right\|^{2}+k_{v_{1}}\left\|v_{13}\right\|^{2}\right. \\
& \left.+k_{v_{2}}\left\|v_{23}\right\|^{2}\right)
\end{aligned}
$$

Thus we have

$$
\dot{v} \leq 0
$$

$\mathcal{V}$ is bounded from below and $\dot{\mathcal{V}} \leq 0$, which implies that $\lim _{t \rightarrow \infty} \mathcal{V}(t)$ exists by monotonicity. To apply La Salle's invariance principle, we construct

$$
\begin{aligned}
\mathcal{K}= & \left\{\left(\mathcal{X}, \Omega_{1}, \Omega_{2}, \xi_{1}, \xi_{2}, \zeta_{1}, \zeta_{2}\right) \in S O(3) \times \mathbb{R}^{6} \times \mathbb{R}^{12} \mid\right. \\
& \mathcal{V}\left(\mathcal{X}, \Omega_{1}, \Omega_{2}, \xi_{1}, \xi_{2}, \zeta_{1}, \zeta_{2}\right) \leq \\
& \left.\mathcal{V}\left(\mathcal{X}(0), \Omega_{1}(0), \Omega_{2}(0), \xi_{1}(0), \xi_{2}(0), \zeta_{1}(0), \zeta_{2}(0)\right)\right\}
\end{aligned}
$$

$\mathcal{K}$ forms a compact invariant set in $S O(3) \times \mathbb{R}^{6} \times$ $\mathbb{R}^{12}$. Now by La Salle's invariance principle, system dynamics converge asymptotically to the largest positively invariant set in subset of $\mathcal{K}$ where $\dot{\mathcal{V}}=0$.

$$
\dot{\mathcal{V}}^{-1}(0)=\left\{\left(\mathcal{X}, 0,0, \xi_{1}, \xi_{2}, 0,0\right) \in \mathcal{K}\right\}
$$

Largest invariant set in $\dot{\mathcal{V}}^{-1}(0)$, can be calculated by equating the control inputs to be zero. For the torque inputs, we find that torque terms vanish at the critical points of $\Psi_{1}$. This gives us $\mathcal{X} \in \mathcal{N} \bigcup\{I\}$ Now from the position control equations we have

$$
k_{d_{1}}\left(d_{12}-d_{12}^{s}\right) l_{12}+k_{d_{2}}\left(d_{13}-d_{13}^{s}\right) l_{13}=0
$$

Since the three spacecraft are assumed to be noncollinear at all times, $l_{13}$ and $l_{12}$ are linearly independent. Hence we get that $d_{12}-d_{12}^{s}=0$, and $d_{13}-$ $d_{13}^{s}=0$. That is $d_{12}=d_{12}^{s}$ and $d_{13}=d_{13}^{s}$.

$$
k_{d_{1}}\left(d_{12}-d_{12}^{s}\right) l_{21}+k_{d_{2}}\left(d_{23}-d_{23}^{s}\right) l_{23}=0
$$

We already have $d_{12}-d_{12}^{s}=0$, and now we have $d_{23}=d_{23}^{s}$. Thus the largest invariant set in $\dot{\mathcal{V}}^{-1}(0)$ is given by

$$
\begin{gathered}
\overline{\mathcal{M}}=\left\{\left(\mathcal{X}, \Omega_{1}, \Omega_{2}, \xi_{1}, \xi_{2}, \zeta_{1}, \zeta_{2}\right) \in S O(3) \times \mathbb{R}^{6} \times \mathbb{R}^{12} \mid\right. \\
\mathcal{X} \in I \cup \mathcal{N}, \zeta_{1}=\zeta_{2}=0, \Omega_{1}=\Omega_{2}=0 \\
\left.\left\|\xi_{1}\right\|=d_{13}^{s},\left\|\xi_{2}\right\|=d_{23}^{s},\left\|\xi_{1}-\xi_{2}\right\|=d_{12}^{s}\right\}
\end{gathered}
$$

from (48)-(50), we can see that if transformed variables converges to asymptotically to $\overline{\mathcal{M}}$, the original dynamics converges to $\mathcal{M}_{1} \cup \mathcal{M}_{2}$. (ii) For all the undesired equilibrium conditions, we have $\Psi_{1}=4$. The value of $\gamma$ is chosen so that

$$
\mathcal{V}(0) \leq 4 k_{1}
$$

Since $\dot{\mathcal{V}} \leq 0$, we have

$$
0 \leq \mathcal{V}(t)<\mathcal{V}(0)<4 k_{1}
$$

This guarantees that the undesired equilibrium configuration is avoided and system dynamics converge to desired configuration $\mathcal{M}_{1}$. As $\gamma \rightarrow 0$, our region of attraction increases to include almost the entire state space with almost semi-global convergence to $\mathcal{M}_{1}$.

(iii) Define

$$
\mathcal{W}=4 k_{1}-\mathcal{V}
$$

At the undesired configuration $\mathcal{M}_{2}$, we have $\mathcal{W}=$ 0 .Now we can choose an arbitrarily close region to $\mathcal{M}_{2}$ where $\Omega_{1}$ or $\Omega_{2} \neq 0$ and the function $\mathcal{W}>$ 0 . Now $\dot{\mathcal{W}}=-\dot{\mathcal{V}}>0$ (strictly greater than zero because we chose $\Omega_{1}$ or $\Omega_{2} \neq 0$ ). Thus there exists at any arbitrarily small neighbourhood of the undesired equilibrium, a solution trajectory that will escape, which gives that undesired equilibrium is unstable (Khalil [1996], Theorem 3.3).

\section{SIMULATION RESULTS}

Numerical simulations are carried out with following initial conditions. Moments of inertia of the two spacecraft are taken to be $J_{1}=J_{2}=\operatorname{diag}[2,3,5] \mathrm{Nm}^{2}$. Let $a=$ $\frac{1}{\sqrt{14}}[1,2,3]^{\top}$, and $\grave{a}=\frac{1}{\sqrt{3}}[1,1,-1]^{\top}$.

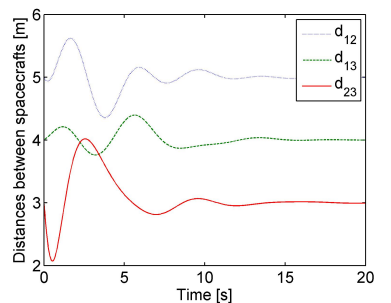

(a) Distance between the spacecrafts $d_{12}, d_{13}$ and $d_{23}$ with time



(b) Norm square of velocity errors $\left\|v_{1}-v_{3}\right\|^{2}$ and $\left\|v_{2}-v_{3}\right\|^{2}$

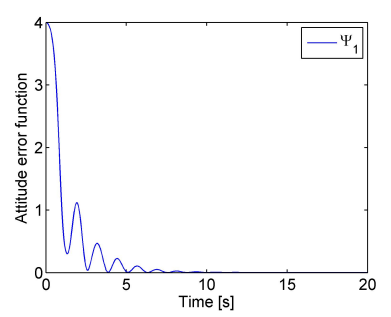

(c) Attitude alignment error function, $\Psi_{1}$ with time

Fig. 1. Distance, velocity errors, $\Psi_{1}$, with respect to time.

Initial conditions are chosen to be close to undesired equilibrium configuration $\mathcal{M}_{2}$. Leader spacecraft is assumed to be at origin initially, $r_{3}(0)=\left[\begin{array}{lll}0 & 0 & 0\end{array}\right]^{\top}$, with velocity $v_{3}(0)=\frac{5}{\sqrt{34}}[3,3,4]^{\top}$. For spacecraft $1, r_{1}(0)=r_{3}(0)+4 \grave{a}$, $v_{1}(0)=\frac{5}{\sqrt{98}}[3,5,8]^{\top} \mathrm{m} / \mathrm{s}, R_{1}(0)=\exp (\pi \hat{a}), \Omega_{1}(0)=$ $[2,-0.1,0.5]^{\top} \mathrm{rad} / \mathrm{s}$, where exp is the matrix exponential. Initial conditions for spacecraft $2, r_{2}(0)=r_{3}(0)+4(a)$, 


\section{CONFIDENTIAL. Limited circulation. For review only.}

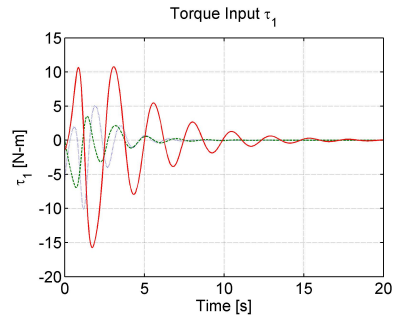

(a) Torque Input for spacecraft

Fig. 2. The torque inputs applied to the two spacecraft. (First component: dotted, second component: dashed, third component: solid)

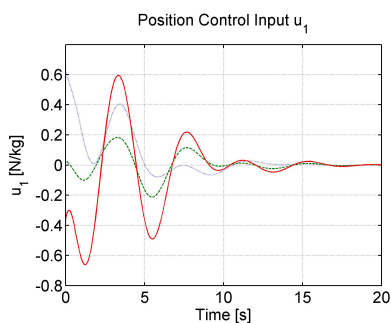

(a) Position Control Input for spacecraft 1

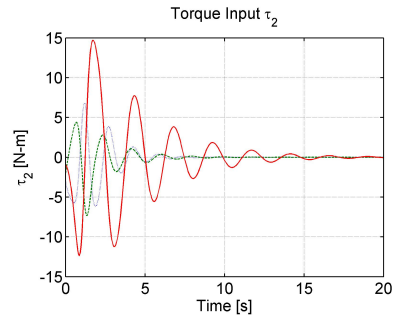

(b) Torque Input for spacecraft
Fig. 3. The position control inputs applied to the two spacecraft. (First component: dotted, second component: dashed, third component: solid)

$v_{2}(0)=\frac{5}{\sqrt{161}}[-5,5,11]^{\top} \mathrm{m} / \mathrm{s}, \Omega_{2}(0)=[1,0.7,0.3]^{\top} \mathrm{rad} / \mathrm{s}$ and $R_{2}(0)=\exp (0.99 \pi \hat{a})$.

The desired distances be $d_{12}^{s}=5 \mathrm{~m}, d_{13}^{s}=4 \mathrm{~m}$, and $d_{23}^{s}=$ $3 \mathrm{~m}$. The control gains are chosen to be $k_{\Omega_{1}}=k_{\Omega_{2}}=3$ $k_{v_{1}}=k_{v_{2}}=0.6, k_{1}=0.7, k_{d_{1}}=k_{d_{2}}=k_{d_{3}}=1$, and $\gamma=\frac{1}{12}$.

Figures in (1) shows distance between satellites achieving desired values and velocity and attitude synchronisation error going to zero. The control inputs are shown in figures (2). Figure (3) shows position control inputs applied to the spacecraft 1 and spacecraft 2 .

\section{CONCLUSION}

In this paper a new combined attitude position control law that achieves attitude synchronization and formation keeping using LOS measurements is proposed. By combining a classical attitude determination scheme and more recent geometric control results, we have improved upon the result by Lee [2012]. The attitude control law obtained in terms of LOS vectors shows synchronization even in presence of position dynamics. The proposed attitude and position control scheme is proven to asymptotically stabilize the desired equilibrium configuration almost semiglobally.

This result can be extended in several ways. The control can be easily modified to achieve any given relative attitude between the two spacecrafts. The collinearity assumption on the spacecraft positions requires more rigorous analysis which will be pursued as part of a future work.

\section{REFERENCES}

D.C. Woffinden and D.K. Geller Observability criteria for angles-only navigation in IEEE Transactions on Aerospace and Electronic Systems, volume 45, number 3, pages 1194-1208, 2009.

P. Gurfill and D. Mishne Cyclic spacecraft formations: relative motion control using line-of-sight measurements only in AIAA Journal of guidance, control, and dynamics, volume 30, number 1, pages 214-226, 2007.

M.S. Andrle and J.L. Crassidis and R. Linares et al Deterministic relative attitude determination of threevehicle formations in AIAA Journal of Guidance, Control, and Dynamics, volume 32, number 4, pages 1077-1088, 2009.

T. Lee. Relative attitude control of two spacecraft on SO (3) using line-of-sight observations. in Proceedings of the IEEE American Control Conference (ACC), pages 167-172, 2012.

R. Warier and A. Sinha LOS Based Attitude Alignment Of Two Spscecraft In Formation in 5th International Conference on Spacecraft Formation Flying Missions and Technologies, Munich, 2013.

J.R. Wertz. Spacecraft attitude determination and control volume 73, Springer, 1978.

N. Chaturvedi, A. K. Sanyal, and N. H. McClamroch, Rigid-body attitude control: Using rotational matrices for continuous, singularityfree control laws IEEE Control Systems Magazine, pages 30-51, June 2011.

A.Sarlette and R.Sepulchre and N.E.Leonard Autonomous rigid body attitude synchronization Automatica,Elsevier, volume 45, number 2, pages 572-577, 2009.

S. Nair and N.E. Leonard Stable synchronization of rigid body networks in Networks and Heterogeneous Media, volume 2, number 4, pages 597, 2007.

A. Sarlette Geometry and symmetries in coordination control PhD Thesis, Université de Liège, 2009.

H.K. Khalil Nonlinear systems. Prentice Hall, 2nd edition, 1996.

T.H.Wu and B. Flewelling, and F. Leve and T. Lee. Spacecraft relative attitude formation tracking on SO (3) based on line-of-sight measurements in Proceedings of the IEEE American Control Conference (ACC), pages 4820-4825, 2013.

D.P.Scharf and F.Y.Hadaegh and S.R.Ploen A Survey of Spacecraft Formation Flying Guidance and Control(Part I): Guidance. in Proceedings of the IEEE American Control Conference, Piscataway, NJ, pages 1733-1739. June 2003.

D.P.Scharf and F.Y.Hadaegh and S.R.Ploen A Survey of Spacecraft Formation Flying Guidance and Control(Part II): Control. in Proceedings of the IEEE American Control Conference, volume 4, pages 2976-2985, 2004. 